\title{
DEVELOPMENT OF AN ARTERIAL APPLANATION TONOMETER FOR DETECTING ARTERIAL BLOOD PRESSURE AND VOLUME
}

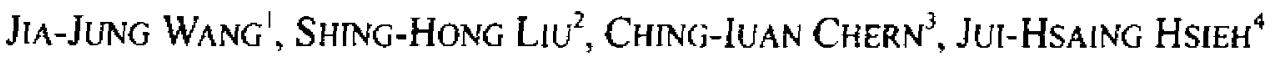 \\ 'Department of Biomedical Engineering, I-Shou University, Taiwan \\ ${ }^{2}$ Department of Biomedical Engineering, Yuanpei University of Science and \\ Technology, Taiwan \\ ${ }^{3}$ Department of Cardiology, Hsin Chu Hospital, Taiwan \\ ${ }^{4}$ Department of Biomedical Engineering, Chung Yuan Christian University, Taiwan
}

\begin{abstract}
Traditional arterial tonometry permits noninvasive and continuous recording of the arterial pressure waveform. by applanating a superficial artery supported by a bone. In the paper, we present an arterial tonometer to simultaneously register the blood pressure waveform and the arterial time-varying volume. The tonometer consisted mainly of a chamber filled with a conductive fluid, a flexible diphragm in touch with an artery, and a pressure sensor used to detect the underlying arterial pressure. In addition, four electrodes were in parallel diposed in the chamber, two of them were triggered with a constant-current source, and the voltage difference between the other two inner electrodes was assocated with the amount of change in the arterial volume. The pressure calibration curve performed with a mercury sphygmomanometer showed a fairly linear relationship $(r=0.998)$ between the tonometer's chamber pressure and the voltage output of the pressure-sensing circuit. The volume calibration was carried out with vessel-like cylinders of various diameters and a linear relationship ( $r=0.884)$ of the change in vessel volume to the voltage output of the volume-sensing circuit was obtained. Clinical testing results revealed that the noninvasive blood pressure measurement with the tonometer was appreciably consistent with the invasive measurement with the catheter-tipped pressure transducer. In summary, the arterial applanation tonometer developed may be used to reliably determine the full arterial blood pressure waveform and the change in the arterial volume, and to make the wall compliance assessment of a superficial artery possible.
\end{abstract}

Biomed Eng Appl Basis Comm, 2004(December); 16: 322-330.

Keywords: arterial tonometer, blood pressure, flexible diphragm, compliance, radial artery, pressure measurement.

Received: Apr 11, 2004; Accepted: Oct 20, 2004

Correspondence: Jia-Jung Wang, Ph.D.

Department of Biomedical Engineering,

I-Shou University, 1, Section 1, Hsueh-Cheng Road,

Ta-Hsu Hsiang, Kaohsiung County, Taiwan 84008

E-mail: wangij@isu.edu.tw

\section{INTRODUCTION}

Methods for accurately monitoring arterial blood pressure waveform have been under investigation for many decades [1-4]. Although invasive method can provide the intact pressure waveforms, the trauma and complication caused to the patient make it undersirable 
in many applications. Although the faithful blood pressure measurements can be obtained by this method, the negative effects on the patient may, in many cases, prevail over the benefits of achieving accurate results from such a method [5-6].

Noninvasive methods for the arterial blood pressure measurefment include predominantly the auscultation $[4,7]$, the oscillometry [8-10], and the tonometry $[1,11-14]$. The auscultatory method, based on the Korotkoff sound, is the most extensively used one for monitoring a patient's arterial blood pressure by medical staff. However, this method is noninvasive, it only provides a measurement of systolic and diastolic pressures on an intermittent basis, and it does not provide the entire blood pressure waveform on a continuous basis. Furthermore, employment of this method often yields inaccurate results. Moreover, the rate at which blood pressure can be recorded is restricted by the inflation and deflation rate of the occlusive cuff. Consequently, true beat-tobeat continuous blood pressure monitoring is unlikely by means of this method.

Most of currently commercialized blood pressure monitors apply the oscillometric method to detect a user's blood pressrue in homecare environment. In the method, occlusive cuff pressure oscillations corresponding to vessel volume pulses demonstrate a specific pattern when the occlusive cuff pressure is gradually decreased from above systolic to below diastolic values. The oscillation amplitude and the cuff pressure are then utilized to determine the mean arterial, systolic and diastolic pressures. It is now, on the whole, accepted that a maximum cuff pressure oscillation takes place when the occlusive cuff pressure is the same as the mean arterial pressure of the subject [15-16]. The systolic pressure is the one where the oscillation amplitude is identical with a systolic ratio of the maximum oscillation in the period of the high cuff pressure, while the diastolic pressure is the one where the oscillation amplitude is equal to a diastolic ratio of the maximum oscillation in the period of the low cuff pressure. This method can provide the users with the heart rate and the discrete pressure values, such as the systolic and diastolic pressures. Among different methods for measuring blood pressure, both the auscultation and the oscillometry are the most prevalent at the present time.

A major advantage of using the arterial tonometry is to record continuous blood pressure waveform. In addition to the tonometry, more than a few methods have been proposed in previous literature for measuring entire blood pressur waveform. Those methods include the photoplethysmography [17-18], the optical method $[2,19]$, and the impedance plethysmography $[1,20-21]$.

One type of arterial tonometers incorporates an array of individual transducer elements placed directly on the patient's tissue overlying an artery or blood vessel from which blood pressure is to be determined [22]. The elements directly sense the mechanical forces in the tissue with which each of them is in contact. The elements of the array are dimensioned and spaced apart from each other such that a plurality of these elements are required to envelop the entire diameter or width of the underlying artery. The size of each element is designed to cover only a small fraction of the diameter of the underlying arery. The pressure of the array against the tissue is increased to suitably applanate the underlying artery without producing occlusion. The blood pressure within the artery is then conducted through the vessel wall and the overlying tissue to the transducers.

An obvious drawback to such sensor-array tonometers includes the use of the discrete elements. It has been found that with such tonometers a continuous contour of the tissue stresses under the array is not yielded [7, 12]. As well, in the previous methods no compensation means is provided for the motion artifact that may influence the forces translated to the sensors from the artery. As a result, it is needed to provide an arterial tonometer intended for the true beat-to-beat measurement of arterial blood pressure. Such a tonometer preferably eliminates the requirement for high-resolution sensor technology and has the ability to register the pressure within vessels.

Therefore, the purpose of the study is to make an effort in developing a new arterial tonometer that is capable of not only measuring the subject's blood pressure waveform, but also recording the change in the arterial volume. In addition, the new tonomter is expected to have ability to assess the mechical compliance of the vessel being monitored.

\section{METHODS}

\subsection{Construction of the Tonometer}

An arterial applanation tonometer modified from the previous study was constructed for the determination of arterial blood pressure and change in vessel volume [11]. The arterial tonometer $(\mathrm{W} \times \mathrm{L} \times \mathrm{H}$ $=2.0 \times 2.0 \times 1.5 \mathrm{~cm}$ ) was principally fabricated using acrylic materials (plexiglass), and was miniaturized such that it could be directly placed over a superficial artery, such as a radial artery. As shown in Fig. 1, the arterial tonometer consists of a hollow chamber that allows operators to perfuse certain kind of conductive fluids into it, a flexible diphragm used to directly contact the superficial artery, and a pressure sensor used to detect the underlying arterial pressure. Four electrodes (alloy of gold and copper) were in 
parallel disposed across the tonometer's chamber to detect the variation of the fluid distribution inside the chamber. For the purposes of convenience, there were two outlets connected to the chamber. One was designed to allow the perfusion of the conductive fluid, and the other to get rid of the bubbles that possibly take place during perfusion procedure. Table 1 summarizes the structural characteristics of the proposed tonometer.

When applied, the arterial tonometer was required to make an appropriate contact with a superficial artery through the flexible diaphragm. To achieve this, we utilized currently a strip bandage to immobilize the tonometer such that a maximal swing in output voltage became apparent. To improve the sensitivity of the tonometer, the short axis of the elliptical opening on the cover was required to be centralized over the underlying artery of interest.

The change in the chamber pressure was continuously monitored with a pressure sensor (NPI12 , Lucas, USA) that was proximally connected to the tonometer chamber. The distance between the sensor and the chamber was kept as short as possible, in order to reduce the distortion of the pressure waveform during transmission

\subsection{Tonometric Principle}

In the study, the variation of the arterial volume was assessed using the impedance plethysmography [1]. In this method, a constant-current source was provided and connected to the tow peripheral electrodes. As the current passed through the conductive fluid (here saline) between the two electrodes, a voltage difference between the two inner electrodes was then induced. In addition, the voltage difference was varied with the fluid distribution in the chambe.

The electric resistance, $R$, of a rectangular compartment that corresponds to the space between the two electrodes can be represented by the following equation,

$$
R=\frac{\rho L}{S}
$$

where $\rho$ is the resistivity of the fluid in the compartment, $L$ is the length and $\mathrm{S}$ is the cross-section area of the compartment. Based on the fact that the volume of the compartment, $V_{m l}$, is the product of $L$ and $S$, the resistance can then be alternatively rexpressed as:

$$
R=\frac{\rho S^{2}}{V_{m l}}
$$

$$
\begin{aligned}
& V_{d}=I R \\
& =\frac{\rho I L^{2}}{V_{m l}}
\end{aligned}
$$

Futher, according to Ohm's Law, the voltage difference $V_{d}$ across the compartment can be obtained by

where $I$ is the current uniformly passing through the compartment.

It is clear from (3) that $V_{d}$ is inversely proportional to the compartment volume. That is, as $\rho$ and $\mathrm{L}$ are maintained and a constant-current source is applied, the larger the volume, the smaller the voltage difference between the two inner electrodes.

\subsection{Block Diagram of Arterial Pressure and Volume Measurement}

The arterial tonometer was designed to measure two vascular variables, the arterial pressure and the change in vessel volume. Fig. 2 illustrates the block diagram of the variable measurement that can be essentially divided into the pressure-sensing and volume-sensing circuits. For the volume measurement, a constant-current source generating a $50 \mathrm{kHz}$ sinusoidal waveform was injected into the two outside

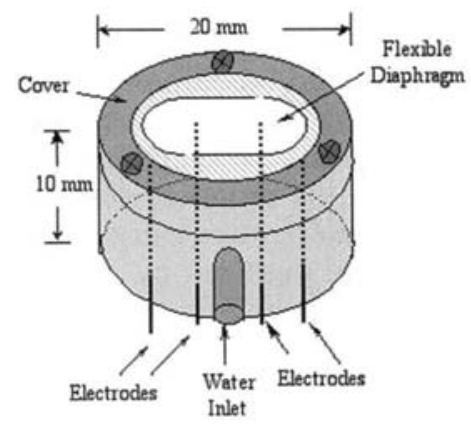

(a)

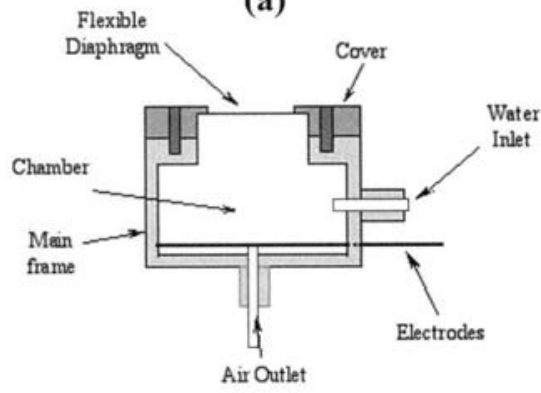

(b)

Fig, 1 (a) Dimensional view and (b) sectional view of the proposed arterial tonometer, showing four wire electrodes arranged in parallet inside the tonometer's chamber and a flexible diaphragm. 
Table 1 Structural features of the proposed arterial tonometer.

\begin{tabular}{ll}
\hline \multicolumn{1}{c}{ Item } & \multicolumn{1}{c}{ Description } \\
\hline Size & $2.0(\mathrm{~W}) \times 2.0(\mathrm{~L}) \times 1.5(\mathrm{H}) \mathrm{cm}$ \\
\hline Weight & $5 \mathrm{~g}($ excluding the pressure sensor $)$ \\
\hline Component separability & $\begin{array}{l}\text { Most components of the tonometer are designed to be separable and the flexible } \\
\text { diaphragm is designed to be interchangeable. }\end{array}$ \\
\hline Coupling medium & A compliant diaphragm is used to couple the varying vessel volume. \\
\hline Fixation & A bandage is used to fasten the tonometer on the wrist by an appropriate force. \\
\hline
\end{tabular}

electrodes, and the induced voltage difference between the two inner electrodes inside the tonometer's chamber was sensed and amplified by means of a differential amplifier. Because the output signal of the differential amplifier had been initially modulated through the sinusoidal waveform with a frequency of $50 \mathrm{kHz}$, it was necessary to demodulate the siganl to obtain the original change in vessel volume. For the arterial pressure measurement, the strain-gauge pressure sensor converted the chamber pressure to a varying resistance. Here, a Wheatstone bridge circuit was designed to yield a zero output when the chamber pressure is equal to the atmospheric pressure. The output signal of the bridge circuit was first high-pass filtered and then properly amplified.

\subsection{Data and Statistic Analysis}

The femoral arterial blood pressure was measured with a catheter-tipped pressure transduce, and the radial arterial blood pressure and vessel volume change were measured with the proposed tonometer for each patient during catheterization surgery. These three analog variables were digitized by a 12 -bit converter, respectively, with a sampling frequency of $200 \mathrm{~Hz}$, and recorded using the MP 100 Manager (BIOPAC System Inc., USA). Subsequent data analysis was performed with a personal computer.

Regresssion analysis of linear relationship between two variables (for instance, invasive pressure and noninvasive pressure) was performed with a software program provided by SigmaPlot (SPSS Inc., USA). Curve-fit methods were applied to find out what kind of function can best delineate the relationship between the arterial pressure and vessel volume [23], if necessary. Correlation coefficients calculated from the linear regression were used to determine how closely the two variables related to.

\section{CALIBRATION}

\subsection{Pressure Calibration}

To calibrate the pressure measurement, the tonometer's chamber and a sphygmomanometer were both connected to an air cuff by means of Y-shaped tubing. The cuff pressre was gradually inflated by an air pump at an incremental rate of $10 \mathrm{~mm} \mathrm{Hg}$ per step. Both the output voltage of the pressure-sensing circuit and the pressure reading of the sphygmomanometer were recorded simultaneously, and the output voltage with respect to the cuff pressure are plotted and shown in Fig. 3. It is obvious that the relationship of the output voltage to the cuff pressure (or the chamber pressure) with an intercept of 1.04 voltage and a slope of 0.02 voltage $/ \mathrm{mmHg}$ is extremely linear, because a high corelation coefficient of 0.998 was obtained.

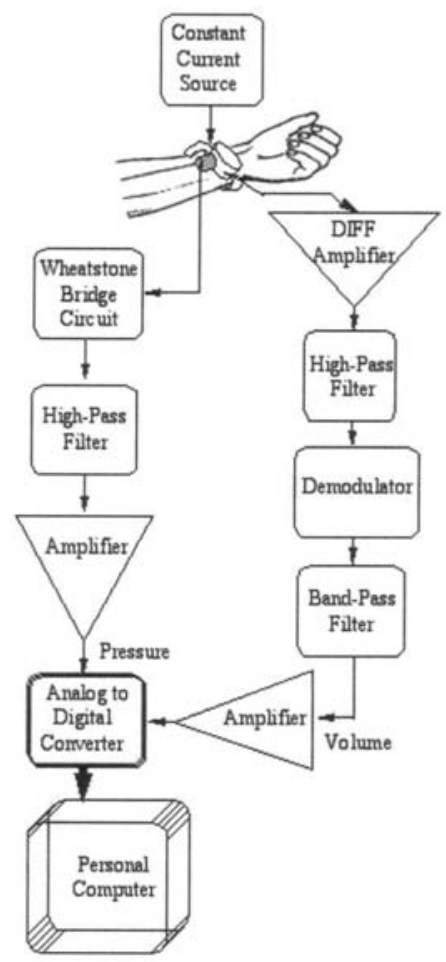

Fig. 2 Block diagram of the arterial pressure and vessel volme measurement. 


\subsection{Volume Calibration}

Fig. 4 demonstrates the schematic diagram to help explain the volume calibration method in the study. As shown in Fig. 4(a), the arterial tonometer is just in touch with the underlying artery, with the assumption that the tissue, mostly skin, between the tonometer's diaphragm and the superficial artery is thin and can be neglected. Fig. 4 (b) illustrates that when the artery is expanded, a part of the artery pushes the flexible diaphragm and intrudes into the tonometer's chamber. In the study, we assumped that the cross-section of the artery remains to be circular as it either dilates or contracts. According to the geometric relationship, the intruded vomue of the artery, Vin (corresponding to the $A B C D$ ), can be determined with the following formula,

$$
V_{\text {in }}=\left(\sqrt{2 r d-d^{2}}-\theta r^{2}\right) L
$$

where $r$ is the radius of the artery, $d$ is the distance from the original position (D) of the diaphragm to the artey wall $(\mathrm{C}), \theta$ is the half of the opening angle that is equal to $\cos ^{-1}[(\mathrm{r}-\mathrm{d}) / \mathrm{d}]$, and $\mathrm{L}$ is the width of the flexible diaphragm as shown in Fig. 2.

We here selected three cylinder tubes having different diameters to simulate the arteries. Once we knowed, by means of a precision meter, the distance between the tube wall and the origial position of the flexible diaphragm, the intruded volume can be readily yielded. By applying different-sized cylindrical tubes, the relationship of the output voltage of the volumesensing circuit to the change in the fluid volume in the chamber is shown in Fig. 5. As we can see, the output voltge varies positively and linearly with the amount of change in vessel volume. Furthermore, all correlation coefficients calculated from these regression lines are $0.968,0.911$ and 0.884 for the tubes with a diameter of $3.95,2.90$ and $1.60 \mathrm{~mm}$, respectively.

\section{TESTING RESULTS}

The feasibity of the arterial tonometer was validated in collaboration with the Department of Cardiology, Hsin Chu Hospital, Taiwan. During catheterization surgery, the blood pressure in the femoral artery was measured with a catheter-tipped pressure transducer, and at the same time, the radial arterial blood pressure and vessel volume change measurements were performed with the proposed tonometer.

Fig. 6 displays one typical recording of arterial variables, including the invasive blood pressure (IBP) waveform of the femoral artery by the catheterization, the noninvasive blood pressure (NIBP) waveform and

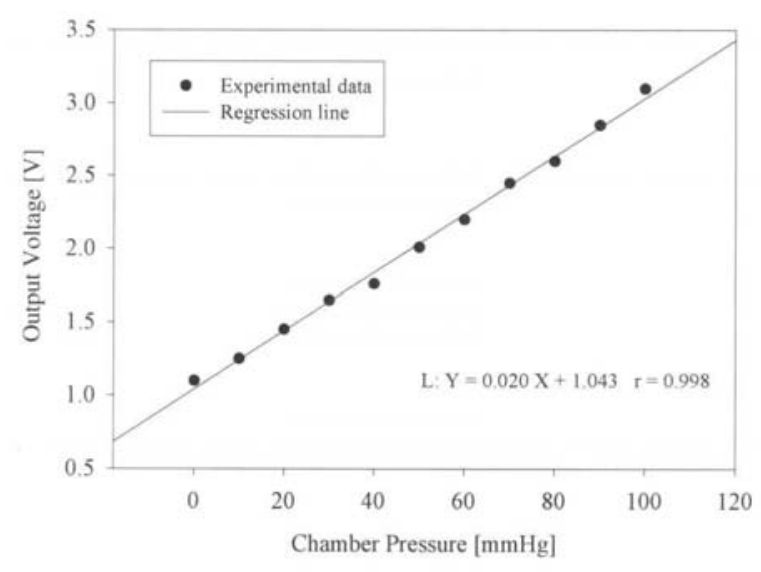

Fig. 3 Relationship between the output voltage of the pressure-sensing circuit and the chamber pressure.

the change in vessel volume of the radial artery by the current tonometer. It is found that there is a small reflection in the descending portion of the radial pressure waveform, while no reflection occurs in the femoral pressure curve. As shown in the figure, the maximum of the femoral blood pressure is up to 190 $\mathrm{mm} \mathrm{Hg}$, but that of the radial pressure is about $160 \mathrm{~mm}$ $\mathrm{Hg}$. Obviously, there is a quite distinction between the two blood pressure maximums, partially due to the different distances from the heart. In Figure 6 (c), the change in vessel volume varies principly with the blood pressure of the radial artery, and incorporates more high frequency components in the volume waveform than the blood pressure curve.

Fig. 7 shows the relationship between the invasive and noninvasive arterial blood pressures. In the ascending portion of the blood pressure, the noninvasive measurement with the arterial tonomerter correlates well to the invasive measurement with the catheterization, and a correlation coefficient of 0.92 is achieved (upper panel). Compared to the ascending portion, a smaller correlation coefficient $(r=0.89)$ between the descending portions of the blood pressures recorded by the proposed tonometer and the cathetertipped pressure sensor is found (middle panel). For comparison of the full blood pressure waveforms with the invasive and noninvasive techniques, a correlation up to 0.932 is obtained (lower panel).

\section{DISCUSSION}

The arterial applanation tonometer developed in the study has been miniaturized to cope with actual applications. It was also validated to measure two 


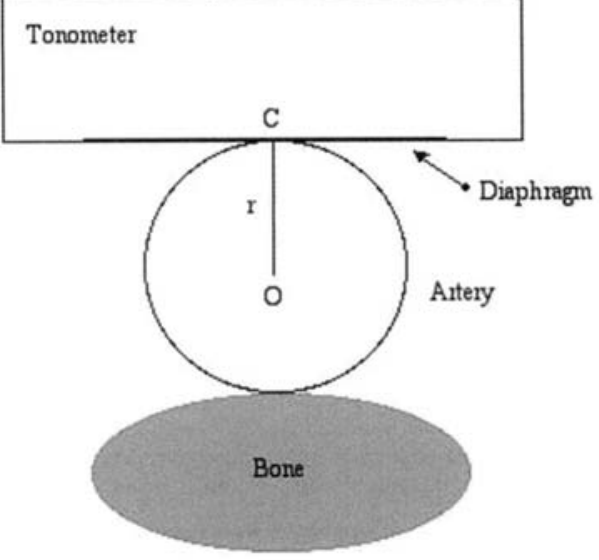

(a)

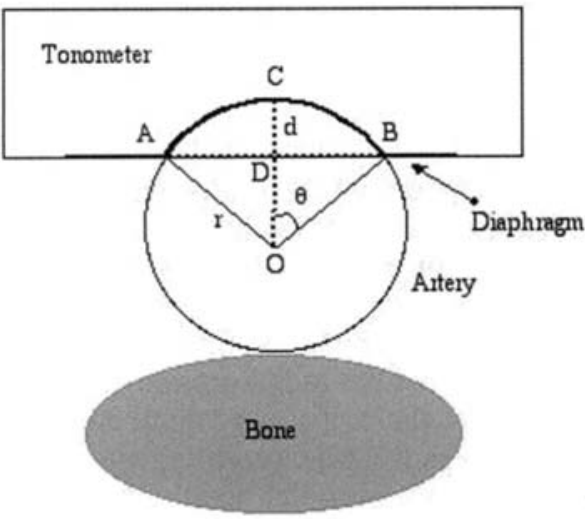

(b)

Fig. 4 Schematic diagram for explaining how to calculate the partial volume of the artery intruding into the tonometer's chamber, when (a) the arterial wall just contacts the flexible diaphragm and (b) the partial arterial volume has intruded into the chamber.

vascular variables, the blood pressure and the vessel volume change, from a superficial radial artery of a patient under catheterization surgery. As we know that most tonometers designed in previous studies merely allow the pressure measurement $[12,24]$. On the other hand, the testing results have shown that the present tonometer permits not only to record the underlying radial blood pressure but also the change in the arterial volume.

To register an accurate arterial pressure waveform with a tonometer, three conditions should be taken into account. They are: (a) the tonometer should be properly immobilized upon the artery of interest, (b) the artery must be superficial to eliminate as much as possible the effects of the subcutaneous tissue between

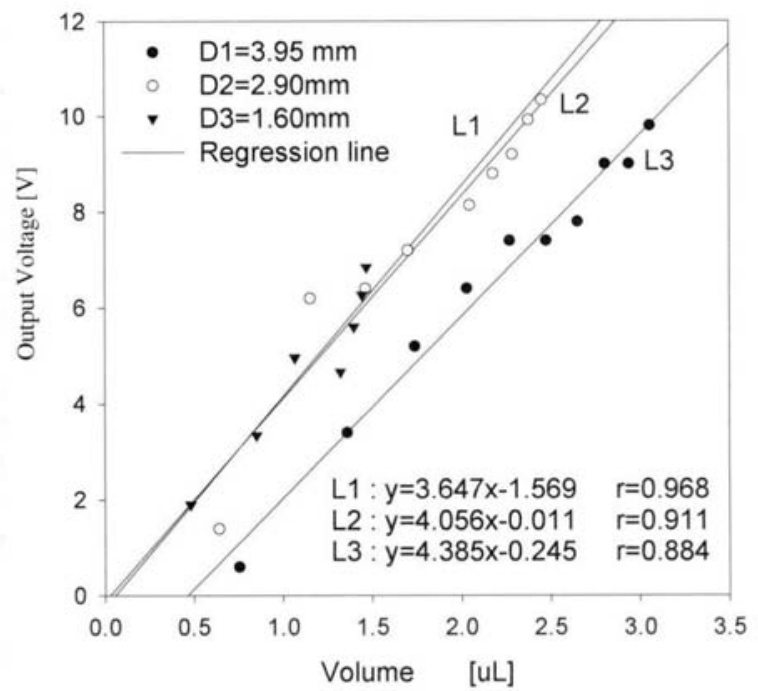

Fig. 5 Relationship between the output voltage of the volume-sensing circuit and the change in the chamber fluid volume, by means of three rubber tubes with different diameters. $D=$ outer diameter of the tube; $L=$ linear regression line; $r=$ correlation coefficient.

the tonometer and the artery, and (c) the vessel should be firmly supported by underlying structure (bone) to allow the application of the tonometer $[1,12]$. In this work, the proposed tonometer is devised to measure the pressure within the radial artery at the wrist. Because the radial artery is superficial and is supported by a bone (radius), it is obvious that the arterial pressure measurement with the current tonometer satisfies completely with the last two conditions.

The force exerted on the tonometer has to adjust according to each subject. Previous investigators have revealed $d$ that different force on the tonometer may lead to different pressure recording, with distortion not only in the amplitude also in the frequency components [13]. Here, we make use of a bandage to immobilize the current arterial tonometer. Before testing or experiment, we have to properly adjust the force exerted on the tonometer to achieve a situation in which maximum pressure amplitude takes place. The recorded values of the full pressure waveform with relative amplitudes can be further processed and calibrated using the data measured with a commercial sphygmomanometer. Then, a whole blood pressure waveform useful in clinical applications may be acquired.

One calibration method used in the previous study is that the authors gradually inject saline into the chamber to find out the relationship between the vessel 


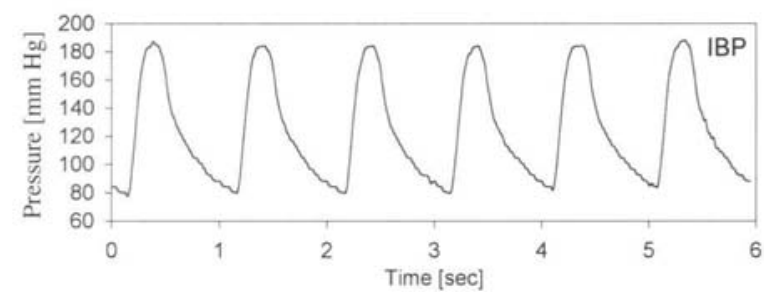

(a)

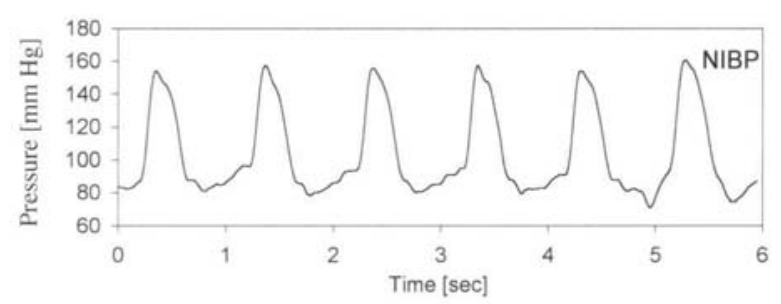

(b)

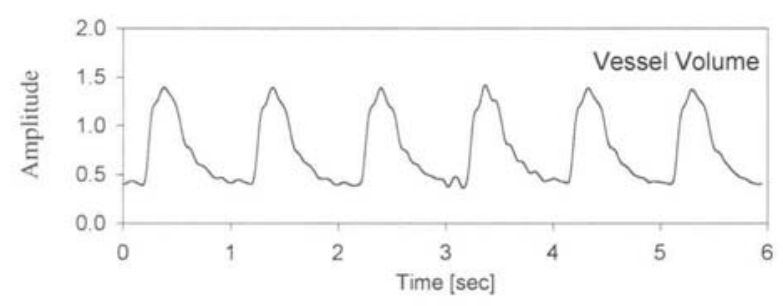

(c)

Fig. 6 Clinical recording. (a) Invasive blood pressure (IBP) from a patinet's femoral artery using a catheter with a tip-sensor; (b) Noninvasive blood pressure (NIBP) and (c) vessel volume from a patent's radial artery using the present tonometer.

volume and the voltage output, resulting in an unreliable outcome [11]. Although the method in the research for the volume calibration of the tonometer is not perfect, it has shown certain degree of progress and improvement. When the measured artery dilates, the expanded volume actually results in a new distribution to the chamber volume. As a consequence, the impedance method used in the work is really to measure the volume redistribution. The calibration performed with this way has demonstrated a linear relationship between the volume redistribution and the output voltage (Fig. 5). With the assumption that the expanded artery is always entirely in touch with the flexible diaphragm, a reliable assessment of change in vessel volume can be achieved if the diameter of the artery of interest is known.

An optimal coupling should be considered in measuring arterial pressure with either the current tonometer or the previous ones. In the present investigation, a flexible diaphragm is required to be compliant enough to directly contact the underlying
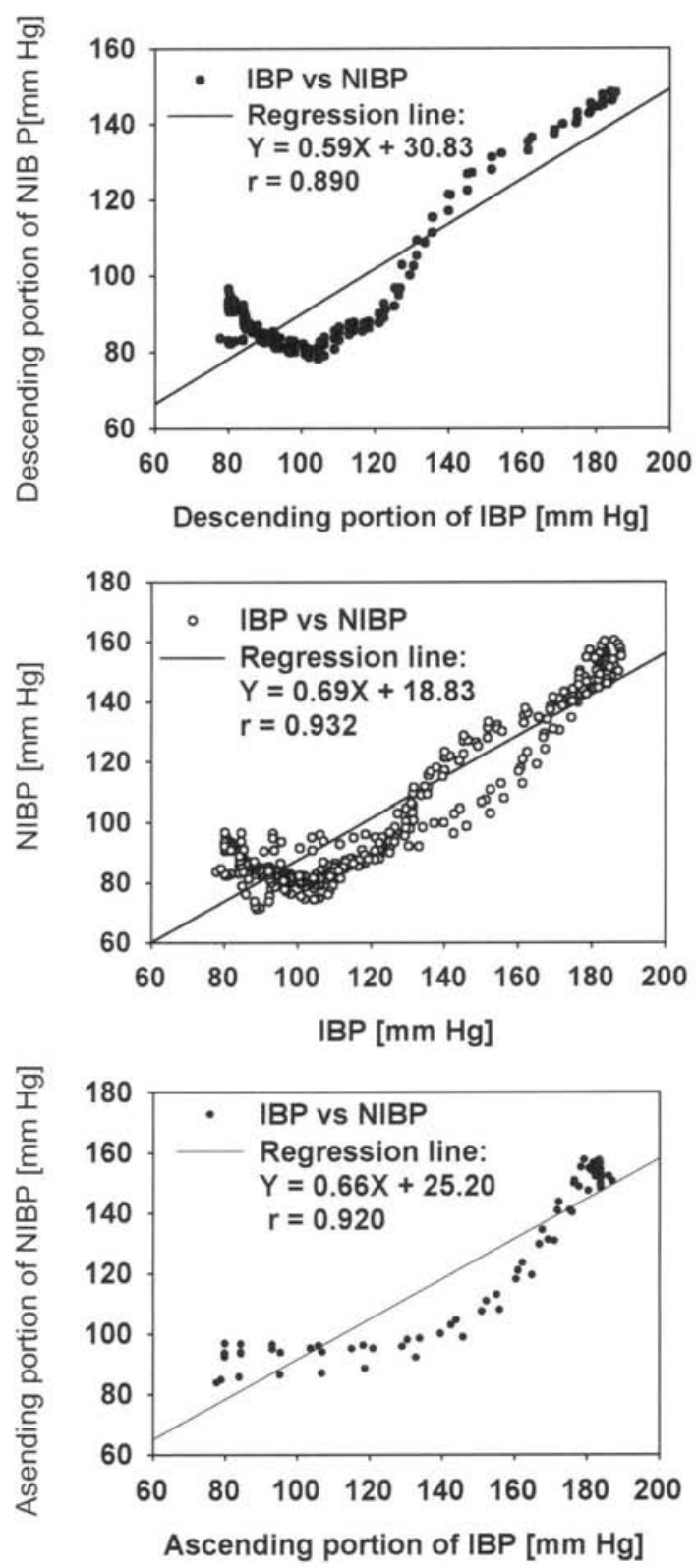

Fig. 7 Comparison between the invasive and noninvasive blood pressures. $r=$ correlation coefficient.

artery. Nevertheless, to approach a least distortion in presssure transmission from the artery to the tonometer, the tonometer's chamber pressure should be varied faithfully with the arterial pressure. In fact, it is fairly diffiult to do that due to the existence of high frequency components in the time-varying pressure. But, if we would like to develolp a sub-optimal coupling condition, the chamber pressure of the tonometer may be maintained at a value equal to the 
mean arterial pressure. This sub-optimal coupling approach has been put into operation in several previous investigations $[16,19]$.

One chief advantage of the current tonometer is that it can be applied to assess the change in an arterial lumen, in addition to the arterial blood pressure measurement. Once the continuous arterial pressure and volume variations are both acquired with the tonometer at the same time, a so-called apparent compliance of the arterial wall can be determined as the ratio of change in vessel volume and change in pressure $[11,24-25]$. This will help the physician diagnose cardiovascular diseases related to the hardening of the entire arterial system or a specific segment of an artery.

A noteworthy drawback to a sensor-array tonometer is the use of the discrete elements. To satisfy with the clinical applications, the size of the array must be miniaturized in order that all sensor elements can be accurately placed over an underlying artery. It always takes time to position the sensors in actual usage. Furthermore, no proper compensation process in a sensor-array tonometer is performed for the motion artifact, affecting the forces converted to the sensors. This problem always causes the sensorarray tonometer to be too sensitive to external stimulations. On the other hand, the opening of the flexible diaphragm of the present tonometer is large enough to cover the contact area with an artery, ensuring that the change in the arterial volume can be successfully transmitted into the tonometer's chamber.

\section{CONCLUSION}

We have developed a bi-functional arterial tonometer in the study to measure the arterial pressure as well as the arterial volume change. The arterial tonometer chiefly contained a fluid chamber, a compliant diphragm, a pressure sensor and four electrodes. The change in the arterial volume is assessed by the impedance method. The pressure calibration using a mercury sphygmomanometer showed that a reasonably linear relationship existed between the chamber pressure and the voltage output of the pressure-sensing circuit. The volume calibration by using vessel-equivalent cylinders with known diameters displayed that there was a linear relationship of the change in vessel volume to the voltage output of the volume-sensing circuit. Clinical testing results demonstrated that the noninvasive pressure measuremenuch with the proposed tonometer was essentially consistent with the invasive pressuer measurement with a catheter-tipped pressure transducer. In brief, the arterial applanation tonometer developed may be used to dependably record full arterial blood pressure waveform and change in vessel volume, and further to determine the dynamic compliance of a superficial arterial wall.

\section{ACKNOWLEDGEMENT}

This research was in part supported by National Science Council, Taiwan, The Republic of China, under the grants of NSC 92-2218-E-214-008 and NSC 92-2218-E-214-013.

\section{REFERERCES}

1. Drzewiecki G, Melbin J and Noordergraaf A: Arterial tonometry: review and analysis. J Biomechanics 1993; 16: 141-152.

2. $\mathrm{Ma} \mathrm{JG}$ and $\mathrm{Xu} \mathrm{DZ}$ : A miniaturized applanation tonometer. IEEE Trans Biomed Eng 1999; 46: 947. 950.

3. Stein PD and Blick EF: Arterial tonometry for the atraumatic measurement of arterial blood pressure. J Appl Physiol 1970; 30: 593-596.

4. Tungjitkusolmun $S$ : Chapter 8-- Heart and circulation. In: Webster JG, Ed. Bioinstrumentation. John Wiley \& Sons Inc, New Jersey, USA, 2004; 262-302.

5. Pressman GL and Newgard PM: A transducer for the continous external measurement of arterial blood ressure. IEEE Trans Bio-Med Electron 1963; 10: 73-81.

6. Iliev I: Improvements of noninvasive long-term blood pressure recording. J Clin Eng 1996; 392-397.

7. Drzewiecki G: Chap 73-- Noninvasive assessment of arterial blood pressure and mechanics. In: Bronzino JD, Ed.-in-chief, The biomedical engineering handbook. CRC Press Inc, Florida, USA, 1995: 1196-1211.

8. Drzewiecki G, Hood R and Apple H: Theory of oscillometric maximum and the systolic and diastolic detection ratios. Ann Biomed Eng 1994; 22: 88-96.

9. Baker PD, Westenskopw DR and Kuck K: Theoretical analysis of non-invasive oscillometric maximum amplitude algorithm for estimating mean blood pressure. Med Bio Eng Comput 1997; 271 278.

10. Lin C-T, Liu S-H, Wang $\mathrm{J}-\mathrm{J}$ and Wen Z-C: Reduction of interference in oscillometric arterial blood pressure measurement using fuzzy logic. IEEE Trans Biomed Eng 2003; 50: 432-441.

11. Drzewiecki, G, Solanki B, Wang J-J, and Li J K-J: Noninvasive determination of arterial pressure and volume using tonometry. Proc ELECTRO '96 1996; pp: $61-63$. 
12. Matthys $\mathrm{K}$ and Verdonck P: Development and modelling of arterial applanation tonometry: a review. Technol Health Care 2002; 10: 65-76.

13. Eckerle JS: Arterial tonometry. In: Webster JG, Ed.-in-Chief, Encyclopedia of medical devices and instrumentation. John Wiley \& Sons Inc, New York, USA, 1988; 2770-2776.

14. Saba PS, Cavallini C, Scorzoni D, Longhini C, Pini $\mathrm{R}$ and Ganau A: Arterial tonometry: principles and clinical applications in hypertension. High Blood Press 1996; 5: 241-250.

15. Bansal V: The mechanics of the occlusive cuff and a flexible diaphragm tonometer. Thesis. Rutgers University, New Jersey, USA, 1994.

16. Wang J-J, Liu S-H, Lin C-T and Hsieh J-H: Modeling the arterial unloaded situation in oscillometric blood pressure waveform measurement using fuzzy logic control. J Med Bio Eng 2001; 21: 93-104.

17. Schnall RP, Gavriely N, Lewkowicz S and Palti Y: A rapid noninvasive blood pressure measurement method for discrete value and full waveform determination. J Appl Physiol 1996; 80: 307-314.

18. Nakagawara $M$ and Yamakoshi $K$ : A portable instrument for non-invasive monitoring of beat-bybeat cardiovascular haemoynamic parameters based on the volume-compensation and electricaladmittance method. Med Biol Eng Comput 2000; 38: 17-25.
19. Yamakoshi K-I, Shimazu H and Togawa T: Indirect measurement of instantaneous arterial blood pressure in the human finger by the vascular unloading technique. IEEE Trans Biomed Eng 1980; 27: 150-155.

20. Sugimachi M, Kawada T, Shishido T, Matsumoto $\mathrm{N}$, Alexander $\mathrm{J} \mathrm{Jr}$ and Sunagawa $\mathrm{K}$ : Estimation of arterial mechanical properties from aortic and tonometric arterial pressure waveforms. Methods Inf Med 1997; 36:250-253.

21. Kim DW and Kim SC: Measurement of leg arterial compliance of normal subjects and diabetics using impedance plethysmography. Ann N Y Acad Sci 1999; 873:112-20.

22. Tsai PS and Yucha CB: Noninvasive measurements of central arterial pressure and distensibility by arterial applanation tonometry with a generalized transfer function: implications for nursing. Heart Lung 2001; 30: 437-44.

23. Johnson RA and Bhattacharyya GK: Statistics: Principles and Methods, 4th Ed., John Wiley \& Sons, Inc, New York, USA, 2001: 441-447.

24. Takayuki S, Masanori N, Akiko K, Toshio O and Hiroyoshi T: Accuracy of a continuous blood pressure monitor based on arterial tonometry. Hypertension 1993; 21: 866-874.

25. Shankar $R$ and Webster JG: Noninvasive measurement of compliance of human leg arteries. IEEE Trans Biomed Eng 1991; 38: 62-67. 